\title{
Medicina di Laboratorio 4.0
}

\section{Laboratory Medicine 4.0}

\author{
Piero Cappelletti $^{1}$ (D) Maria Golato $^{2}$
}

Ricevuto: 26 ottobre 2018 / Accettato: 13 novembre 2018 / Pubblicato online: 5 dicembre 2018

(C) Società Italiana di Patologia Clinica e Medicina di Laboratorio 2018

Riassunto La Medicina di Laboratorio 4.0, corrispettivo della Quarta Rivoluzione Industriale, è la fusione di tecnologie fisiche, chimiche, biologiche e informatiche al servizio della sua mission specifica: fornire informazioni (dati, interpretazioni e consulenze) sulla salute dell' essere umano, provenienti dai suoi fluidi e tessuti e ottenuti con tutti i mezzi d'indagine disponibili. I suoi prodromi possono essere trovati nei lavori degli anni '90 e duemila sulla convergenza di tecnologie, l'importanza dell'Information and Communication Technology (ICT), la costruzione di meta-network per Laboratori virtuali e l'innovazione distruttiva indotta da ICT e miniaturizzazione (POCT). Essa si afferma attraverso alcune tendenze principali come: la medicina molecolare con la progressione da genetica a genomica e post-genomica che coniugata con la rivoluzione digitale approda alla diagnostica della Medicina di Precisione; lo sviluppo tecnologico della miniaturizzazione di metodi e strumenti e della diagnostica al punto di necessità del paziente che traguarda ai lab-on-chip e ai biosensori indossabili e impiantabili; la "trasformazione digitale" che conduce, in senso stretto, alla tele-patologia e alla nuova "scienza cellulare" e ai biomarcatori digitali dall'altro. Cambia il mondo della professione: il professionista di laboratorio deve continuare a garantire $\mathrm{i}$ compiti fondanti di ricerca, informazione, qualità e "traslazione", ma con la conoscenza delle nuove tecnologie; il nuovo paziente (e-patient) userà nuovi approcci per l'accesso on line e per risposte più informative, dirette e "virtuali".

Parole chiave Medicina di Laboratorio 4.0 - Medicina di precisione $\cdot$ Point-of-care testing $($ POCT) - Intelligenza artificiale - Trasformazione digitale

\footnotetext{
P. Cappelletti

pie.cappelletti@gmail.com

1 SIPMeL, Castelfranco Veneto, TV, Italia

2 ASL02 Lanciano-Vasto-Chieti, Lanciano, CH, Italia
}

Summary The Laboratory Medicine 4.0 is the corresponding event in Laboratory Medicine of Fourth Industrial Revolution, characterized by fusion of physical, digital and biological worlds, for improving its mission, i.e. to provide information for the health of the human being, examining its body and fluids by a complex set of technical tools. First signals were the works about converging technologies, vertical laboratory meta-network, and disruptive innovation by Information and Communication Technology (ICT) and miniaturization (Point-of-care Testing, POCT). Now, the main trends are the evolution of molecular medicine from genetics to genomics and post-genomics that, coupled with digital revolution, brings to the Precision Medicine; the development of miniaturization of methods and instruments that looks to lab-on-chips and to wearable and implantable biosensors; the digital transformation that allows the telepathology and a new "cellular science" as well as a set of digital biomarkers. The professional world of Laboratory Medicine is challenged: the basic tasks of quality, information, research, and "translation" remain but new technologies should be rapidly integrated in the core curriculum; the e-patient will use new ways for accessing the service and for obtaining quick, precise, and informative answer to his/her clinical question.

Keywords Laboratory Medicine 4.0 - Precision Medicine. Point-of-care Testing (POCT) · Artificial intelligence . Digital transformation

\section{Medicina di Laboratorio 4.0: una definizione}

Non esiste una definizione consolidata di Medicina di Laboratorio 4.0, principalmente per la novità del concetto e dei contenuti di tale locuzione. Tuttavia, poiché esiste una de- 
finizione sufficientemente accettata di Medicina 4.0 (il corrispettivo nella scienza e nella pratica medica della Quarta Rivoluzione Industriale che è caratterizzata dalla fusione di tecnologie fisiche, digitali e biologiche in grado di impattare tutte le discipline mediche e di sfidare l'idea comune di cosa significhi essere umani) [1], possiamo coniarne una su quel modello. La Medicina di Laboratorio 4.0 è, dunque, la fusione di tecnologie fisiche, chimiche, biologiche e informatiche al servizio della sua mission specifica: fornire informazioni (dati, interpretazioni e consulenze) sulla salute dell'essere umano, provenienti dai suoi fluidi e tessuti e ottenuti con tutti i mezzi d'indagine disponibili. Come si può vedere, è scritto nei fondamentali della disciplina (verrebbe da dire nei suoi geni) l'abitudine e la caratteristica dell'utilizzo di molti mezzi d'indagine variegati e complessi fisici, chimici e biologici; l'elemento nuovo è il ruolo centrale dei mezzi informatici e digitali, utilizzati non solo come metodi d'indagine o componenti essenziali delle tecniche strumentali ma, anche o soprattutto, come strumenti di elaborazione, integrazione e sintesi dei dati ottenuti con metodi e tecniche diverse. In effetti, ciò che caratterizza la Medicina di Laboratorio 4.0 è la fusione, non la somma e nemmeno la sinergia, di tecnologie diverse che impatta tutte le sue (sub)discipline e sfida quel concetto stesso di "umano" cui la sua mission fa riferimento.

Recentemente, il termine Medicina di Laboratorio 4.0 si è diffuso all'interno della disciplina. Analytica 2018 [2], la più importante mostra mercato europea del settore, ha affrontato, nel marzo di quest'anno, la crescita e la maturazione di automazione e digitalizzazione nel Laboratorio del futuro in sessioni dal titolo "Analytica 4.0. The future lab \& the lab 4.0". La $2^{\text {a }}$ Conferenza Strategica EFLM (European Federation of Laboratory Medicine) [3], dal titolo "The end of laboratory medicine as we know it? Handling disruption of Laboratory Medicine in digital health", ha discusso nel giugno 2018 anche di "Diagnostics 4.0: the medical laboratory in Digital Health". Ciò testimonia che l'intuizione di SIPMeL, nel 2017, di intitolare il suo $4^{\circ}$ Congresso Nazionale "Patologia e Medicina di Laboratorio 4.0" la colloca sulla cresta dell'onda dell'innovazione e della complessità della Diagnostica di Laboratorio.

\section{Prodromi}

In Medicina di Laboratorio, il concetto della fusione di tecnologie e di ruolo guida dell'informatica è presente da almeno vent'anni. In un profetico lavoro del 1996, intitolato "Converging technologies and their impact on clinical laboratory" [4], Carl Burtis individuava nell'integrazione delle nuove tecnologie di misura, nell' accelerazione digitale (maturazione dei computer secondo la legge di Moore), nella rivoluzione comunicativa legata principalmente a Internet e nelle nuove forme di trasporto che consentono organizzazioni just-in-time il movente dell'incombente rivoluzione nel Laboratorio clinico. Il punto di vista di Burtis era quello dell'organizzazione del Laboratorio e non prevedeva l'arrivo degli smartphone, ma nelle tecnologie emergenti citava l'automazione integrata ed, espressamente, la miniaturizzazione e le metodologie molecolari. Nel $2001 \mathrm{Bru}-$ ce Friedman [5] descriveva una Total Laboratory Solution, una meta-rete verticale di Laboratori integrati tenuti insieme dall'Information and Communication Technology (ICT), per fronteggiare le sfide organizzative ed economiche e quelle delle nuove tecnologie molecolari con l'apertura all'integrazione delle informazioni cliniche e al rapporto con il paziente. E, nel 2010, Chrisopher Price [6] ricordava che Clayton Christensen, il teorico della "disruptive innovation", aveva suggerito che gli strumenti di Point-of-care testing (POCT) e l'ICT costituivano i due esempi di innovazione dirompente/distruttiva nell'ambito del Laboratorio, perché consentono (obbligano) a una Medicina più "precisa", a livello sia diagnostico sia organizzativo, sconvolgendo i modi tradizionali di fornitura del servizio e i ruoli e compiti dei suoi operatori.

\section{La nascita della Medicina di Laboratorio 4.0}

Non esiste, quindi, una data di nascita della Medicina di Laboratorio 4.0, ma piuttosto un progressivo affermarsi di tendenze tecnologiche, tra loro intersecantesi, d'innovazione medica, di automazione strumentale, di miniaturizzazione e di informatizzazione, riferibili principalmente allo sviluppo delle tecniche di biologia molecolare, dei sistemi di analisi al letto del paziente e della digitalizzazione.

Uno snodo essenziale fu certamente l'introduzione durante la prima decade del nuovo millennio nella Medicina molecolare, che già utilizzava analisi degli acidi nucleici basate sull'ibridizzazione (blotting) o sull'amplificazione ( $P O$ lymerase Chain Reaction e tecniche conseguenti), dei microarray (tecnica di ibridizzazione inversa, sviluppata negli anni '90) deputati alla Gene Expression Profile Analysis, strumento allora considerato rivoluzionario per lo studio delle malattie croniche e degenerative complesse, perché fu presto chiaro che la complessità dei dati prodotti (acidi nucleici), delle variabili tecniche (standardizzazione) e delle ipotesi interpretative aveva la necessità di elaborazioni precise, veloci e affidabili ottenibili solo con sistemi informatici di analisi di base dati, sviluppati ancora negli anni ' 80 per lo studio del genoma del fago [7].

I microarray rappresentarono un concreto esempio di fusione tecnologica (ibridizzazione nel senso delle cellule produttrici di anticorpi monoclonali) tra biologia e informatica e la porta per la "genomificazione" della Medicina. Esistevano comunque problemi tipicamente laboratoristici in questa 
rivoluzionaria tecnica: la distinzione tra tessuto "normale" che è tipicamente dinamico e quello "patologico"; l'eterogeneità cellulare e subcellulare; la comparabilità dei risultati legata a differenze di tecnica e assenza di standardizzazione; il problema della conferma dei risultati con metodi diversi e la gestione comune e comprensibile dell'immane mole di dati. La bioinformatica era, dunque, divenuta essenziale. La Medicina genomica, basata fondamentalmente sullo studio dei single nucletide polymorphism (SNP), ha consentito, comunque, una Medicina "personalizzata" basata sulla suscettibilità individuale alla malattia e soprattutto sulla risposta individuale alle terapie (farmacogenetica e farmacogenomica indicate collettivamente come PGx).

A essa è succeduta, secondo Hiroshi Tanaka [8], la Medicina post-genomica (post-genomic omics information) basata sulle "omiche" (proteomica, transcrittomica, metabolonica) in grado di produrre una Medicina "predittiva" grazie alla sotto-classificazione per la prognosi delle malattie e all'identificazione della risposta ai farmaci antitumorali, in quanto l'uso delle high-throughput technologies (HTT) come i microarray e la spettrometria di massa, che si era intanto sviluppata, consente veloci riconoscimenti dei pattern di espressione genica.

Una terza generazione "omica", quella della omics-based system medicine, è dominata dalle HTT come la NGS (next generation sequencing) e dall'elaborazione computazionale e va intesa come comprensione globale delle interrelazioni dei percorsi molecolari distorti, dell'organizzazione gerarchica dei network, dell'auto-mantenimento delle condizioni morbose e della dinamica della malattia, nella consapevolezza che le malattie comuni hanno pattern complessi, non solo genomici o post-genomici, ma coinvolgenti network molecolari orizzontali e verticali nell'organizzazione degli organismi viventi. Di qui la possibilità concreta di una 4Ps medicine (predictive, personalized, preventive, participatory).

Questa progressione è stata possibile grazie allo sviluppo della bioinformatica clinica o traslazionale. Se la Medicina genomica ha utilizzato un approccio informatico basato sulla statistica classica, quella post-genomica sta usando approcci informatici data-driven basati sul data-mining, sul machine learning e sull'explanatory statistics, mentre la Medicina sistemica si affida ad approcci che utilizzano di nuovo ipotesi seppur modellizzate (model-driven) e basate sull'identificazione, simulazione e disegno dei sistemi.

Attraversando le generazioni "omiche", si traguarda l'orizzonte clinico costituito dalla Medicina di Precisione [9]. Essa tende al trattamento e prevenzione delle malattie sulla base della variabilità individuale dei geni, ambiente e stili di vita (personalizzazione) e si basa sulla comprensione deterministica delle malattie, diagnosi di fattori causali, abilità di trattare le cause profonde della malattia, utilizzando strumenti come i database biologici genomici e post-genomici, metodi di caratterizzazione quali "omiche", analisi cellulari e tecnologia "mobile", la bioinformatica e l'intelligenza artificiale. In modo estremamente sintetico si sostiene che la Medicina di Precisione sia la somma della genomificazione e della bioinformatizzazione della medicina, dimenticando che la visione è sistemica e coinvolge altresì i livelli ambientali e degli stili di vita. Tuttavia sono la biologia divenuta "data-intensive", il grande numero di dati disponibili strutturati e no (big data), le nuove possibilità computazionali $\mathrm{e}$ una visione sistemica a consentire il cammino verso la Medicina di Precisione. D'altra parte, la classica "bioinformatica", intesa come un campo interdisciplinare che sviluppa mezzi informatici e computazionali per analizzare e comprendere dati biologici molecolari, infra-molecolari e sopramolecolari, combinando statistica, matematica, ingegneria informatica e scienza dei computer, progressivamente non appare più sufficiente per l'integrazione di enormi quantità di dati, di livelli biologici complessi e di numerosissime possibilità di scelta (big data). Oggi l'utilizzo dell'intelligenza artificiale è divenuta un elemento essenziale per lo sviluppo della Medicina di Precisione diagnostica, come per esempio nell'interpretazione di pannelli biochimici complessi, di profili metabolici o dei risultati della biopsia liquida, tramite "sistemi esperti", "reti neurali" o applicazioni di "machine learning". Per esempio, il sistema MSK-IMPACT (Integrated Mutation Profiling of Actionable Cancer Targets) [10] è il primo pannello approvato dalla FDA per la profilatura de tumori, in grado di analizzare, nel tessuto tumorale e/o nel sangue, le mutazioni di 468 geni implicati nei diversi tipi di cancro e di identificare trattamenti efficaci (targeted therapy), al di là della tipizzazione istologica e sulla base delle mutazioni somatiche e dell'instabilità satellite individuate.

Lo sviluppo dei POCT è stato un altro big driver della fusione di tecnologie nel Laboratorio clinico. In origine, le più semplici applicazioni si basavano sul principio del lateral flow immunoassay (LFIA) che unificava tecniche fisiche di trasporto fluidico, tecniche biologiche di riconoscimento dell'analita, tecniche chimiche di rivelazione e, talora, tecniche fisiche di lettura della positività/negatività del risultato. Una seconda generazione di strumenti è stata in grado di identificare antigeni tramite immunocromatografa o l'applicazione di tecniche molecolari [11]. La famiglia degli strumenti GeneXpert, per esempio, è un sistema in grado di automatizzare e integrare tutti i passaggi di un'analisi molecolare: preparazione del campione, amplificazione isotermica del DNA, rivelazione tramite PCR-NAT, lettura in fluorescenza. L'uso di diversi composti fluorescenti consente la ricerca contemporanea di target multipli nello stesso campione; un sistema esperto definisce i risultati. Le prospettive coinvolgono le nanotecnologie con tecniche di "local heating" di nanoparticelle, che sostituiscono i thermal cycler, seguite da un' amplificazione laser-PCR e una lettura in fluorescenza, che risolvono molti problemi analitici e preanalitici, oppure con microsensori nanostrutturati in grado di 
"leggere" macromolecole di acidi nucleici senza i passaggi dell' amplificazione con PCR. Importanti campi si aprono per le tecnologie non-NAT con microarray di proteomica e peptidica, nuovi oggetti di ricerca (aptameri, scaffold, anticaline), miglioramenti nell'origine e trasmissione del segnale (ottica label-free, magneto-resistenza), per nuovi bioMEMS (Biological Microelectromechanical Systems), per applicazioni diagnostiche legate a smartphone (per esempio, Phone Mobile Microscopy) e per innovativi modi di produrre e interpretare i risultati sulla base di algoritmi integrati e/o di applicazioni di comunicazione Wi-Fi [12].

L'evoluzione è la costruzione di lab-on-chip, dispositivi che integrano funzioni multiple di laboratorio in un singolo microprocessore di computer, costituito da una piastrina di silicio con circuiti integrati che va da pochi millimetri a qualche centimetro quadrato di grandezza ed è capace di trattare volumi di fluidi $10^{-9} / 10^{-18}$. La tecnologia biologica coinvolta è quella molecolare (genomica e proteomica), ma anche l'analisi cellulare, mentre la tecnologia fisica è quella della microfluidica (liquidi in volumi di nanolitri o picolitri in canali di diecine/centinaia di micrometri). La microfluidica, la chip technology, le tecniche fotolitografiche e le rivelazioni elettrochimiche, immunometriche e molecolari hanno consentito la miniaturizzazione dei sistemi e la possibilità di numerose determinazioni parallele nella cosiddetta "micro-total analysis systems" ( $\mu$ TAS). Le possibilità offerte dall'informatica e dal networking consentono la lettura dei segnali e l'interpretazione dei risultati non necessariamente nel luogo dell'esecuzione del test [11].

L'attuale frontiera dei biosensori prevede la possibilità di misura continua nel tessuto sottocutaneo (con un passaggio di microdialisi) o tramite applicazioni dirette sulla pelle ("tatuaggi"; stampe 3D) per giungere all'automazione dell' organo artificiale, come già avvenuto per il pancreas [13].

Per digitalizzazione s'intende, stricto sensu, il processo di conversione di misure di fenomeni fisici che ne determina il passaggio dal campo dei valori continui a quello dei valori discreti, sintetizzato nella transizione dal mondo dell'analogico a quello del digitale. In Medicina di Laboratorio esempi operativi sono dati dagli strumenti di digitalizzazione delle immagini morfologiche (di sangue periferico, di preparati citologici o istologici) per una loro visione a distanza, per un confronto virtuale con modelli patognomonici o esperti in network, per l'interpretazione diagnostica tramite sistemi di riconoscimento di pattern o algoritmici. Tuttavia la "Digital Pathology" [14] non si limita alla scannerizzazione di un vetrino virtuale, ma evolve verso l'integrazione e la condivisione delle immagini virtuali e di altri dati digitali di laboratorio (barcoding, electronic specimen tracking, workflow management, digital dictation, reporting synopsis), per una sintesi di qualità del total testing process istopatologico o citologico, e clinici per l'interpretazione diagnostica e con un possibile futuro visionario di "cellular science" inserita in una nuova transdisciplina. Secondo alcuni autori [15], si tratta di un esempio di transizione dalla "digitation" (trasformazione in dati digitali), alla "digitalization" (sistemi autonomi di elaborazione di dati digitali) e quindi alla "digital transformation" rappresentata da un ambiente caratterizzato dall'interazione di molteplici sistemi digitali tra loro e con sistemi non digitali (umani). I sei pilastri della trasformazione digitale sono automazione, informatizzazione, dematerializzazione, virtualizzazione, "cloud computing" e "mobile". Il ruolo della telefonia mobile, o meglio degli smartphone, è sempre più pervasivo anche in Medicina di Laboratorio, dalle semplici "app" per la gestione diretta di analisi da parte del paziente all' utilizzo tramite applicativi in termini diagnostici (microscopia, determinazioni biochimiche) e al ruolo fondamentale nella wearable diagnostics, per l'integrazione dei dati, la loro condivisione (per esempio, in una cartella clinica digitale) e un primo livello interpretativo come la definizione delle variazioni intra-individuali (reference change value) e suggerimenti diagnostici. I prodotti della diagnostica indossabile sono già stati definiti "biomarcatori digitali". "Digital biomarkers are defined as objective, quantifiable, physiological, and behavioral data that are collected and measured by means of digital devices such as portables, wearables, implantations, or digestibles" [16]. I dati così raccolti sono tipicamente usati per spiegare e/o predire esiti di salute. I biomarcatori digitali rappresentano l'opportunità di catturare dati oggettivi clinicamente significativi e devono presentare tre caratteristiche specifiche: " $a d$ herence" (vanno raccolti con continuità), "agreement" (con i criteri clinici attuali) e "augmentation" (nel senso che devono offrire informazioni al di là di quelle ottenibili con i metodi clinici odierni). Essi potrebbero costituire la base dei futuri studi clinici e del monitoraggio della salute dei pazienti.

La digitalizzazione diffusa e la possibilità d'interconnessione con dati non strutturati generano i cosiddetti Big Data [17], un insieme di dati così numeroso e complesso da essere ingestibile con gli strumenti tradizionali, compreso il cervello umano. I Big Data vengono spesso rappresentati dal "modello 3V" di Gartner: grande volume della massa di dati, grande velocità di flusso dei dati, grande varietà di tipologia di dati. Qualcuno aggiunge altre $2 \mathrm{~V}$ : veracity (legata al grado di incertezza dei dati) e valore degli stessi. In Medicina, e in Medicina di Laboratorio, i Big Data sono ormai di casa sia per la ricerca scientifica (i nuovi "basket trial" o "umbrella trial" per i marcatori tumorali complessi) sia per la Medicina applicata (prevalenze e tendenze di malattia, fattori di rischio, rapporto genoma e fenoma, efficacia ed efficienza di metodi e strumenti). Essi possono essere usati anche per la gestione, epidemiologicamente corretta, delle linee d'azione del Laboratorio in termini di appropriatezza 
diagnostica ed efficacia clinica, consentendo in tempo reale la valutazione e il monitoraggio delle abitudini prescrittive e dei fattori interconnessi (RM Dorizzi, comunicazione personale).

\section{Le prospettive}

Le direttrici di penetrazione della Medicina di Laboratorio 4.0 nella realtà diagnostica che stiamo vivendo possono essere, quindi, condensate nel processo di convergenza delle tecnologie che sta realizzando un'autentica "fusione di tecnologie", in particolare nel campo della diagnostica al punto del paziente (near patient testing/point of need testing) e nella Medicina di Precisione, e nella pervasiva trasformazione digitale, che coinvolge informatizzazione, connettività, "mobile technology", utilizzo dell'intelligenza artificiale e la diffusione dell'utilizzo dei Big Data per i sistemi sanitari (epidemiologia, appropriatezza, controllo dell'efficacia/efficienza). Il passo della loro avanzata e il successo di ogni singola innovazione non sono facili da predire, perché molte sono state le profezie non avverate nella storia della Medicina di Laboratorio, come ci ha ricordato qualche anno fa Larry Kricka et al [18]. Tuttavia, lo stesso Kricka nell' anno in corso nella sua lettura introduttiva alla Seconda Conferenza Strategica di EFLM [3] "History of disruptions in Laboratory Medicine: what have we learned from predictions?" enumerava le predizioni avverate: penetrazione delle nanotecnologie (eccetto i nanorobot), pervasività degli smartphone, diagnostica indossabile, riorganizzazione geografica dei Laboratori, aumento nella richiesta di test farmacogenetici, diffusioni di individui con sequenziamento del proprio genoma. Il futuro è già qui.

Le tendenze della Medicina di Laboratorio 4.0 stanno determinando, secondo la lezione di Eric Topol [19], l'emersione di un nuovo paziente autonomo e interconnesso - homo digitus o e-patient - che trova strade alternative alla medicina tradizionale, affidandosi ai vari dottori Google, Smartphone, Wearable, IoT (Internet of Things) sia per la gestione organizzativa della sua salute sia per la soluzione clinica delle sue patologie. Ciò pone un problema importante per la Medicina di Laboratorio: i modi e i contenuti del rapporto diretto con l'e-patient. Almeno due aspetti sono immediatamente percepibili: le forme dell'accessibilità e quelle della risposta. Per quanto riguarda le prime, sono prevedibili forme di accesso on line, caratterizzate dalla scelta del fornitore di servizio sulla base di parametri conosciuti di qualità percepita e intrinseca, simili a quelle funzionanti per altri servizi (per esempio, booking.com). La scelta delle caratteristiche da segnalare e l'impatto user-friendly saranno elementi essenziali come fattori critici di successo. Si possono concepire altri approcci più diretti, come le "piattaforme conversazionali", strumenti simili agli assistenti vocali di iPhone o di Google che accolgono e indirizzano i pazienti in forma vocale (per esempio, la First Aid App della Mayo Clinic). Per quanto riguarda le forme di risposta, alcuni autori suggeriscono formati visivamente più comprensibili delle classiche risposte numeriche con l'indicazione a colori della posizione dei risultati rispetto agli intervalli di riferimento popolazionistici o ai propri valori di base, altri insistono su suggerimenti automatici via mobile con sms o direttamente su app in relazione a risultati di allarme o deviazioni significative dalla linea di base, altri infine puntano maggiormente sulle iperconnettività attraverso e-mail, chat e messaggi scritti e vocali per un più coinvolgente contatto con il paziente. Tuttavia, queste forme di collegamento sono completamente o preponderatamente automatiche e/o basate su sistemi esperti o di machine learning.

Infine, la professione ne sarà pesantemente condizionata. Molte attività diagnostiche continueranno a migrare all'esterno dei Laboratori e presso pazienti fortemente $\mathrm{em}$ powered. Le attività in Laboratorio saranno di elevata specializzazione. La conoscenza delle tecnologie innovative e la capacità di comprendere la trasformazione digitale, l'intelligenza artificiale e i Big Data saranno fattori critici di successo. Un rapporto nuovo semi-digitale e semi-umano con i richiedenti esami, siano essi medici o pazienti, sarà gestibile con tecnologie mobili e disponibilità empatiche $\mathrm{e}$ open-minded.

Restano essenziali, seppure trasformate, le attività che traguardano la garanzia della qualità, il knowledge management, la ricerca traslazionale (applicazione delle innovazioni ed evidenze nella pratica clinica) e "trasformazionale" (applicazione delle innovazioni ed evidenze nell'organizzazione del servizio) e la generazione di "evidenze" utili all'uso dei nuovi test e all'impatto assistenziale del nuovo Laboratorio, ma anche alla strutturazione e finanziamento delle organizzazioni [20].

\section{Conflitti di interesse Nessuno.}

Studi condotti su esseri umani e animali Per questo tipo di studio non è richiesto l'inserimento di alcuna dichiarazione relativa agli studi effettuati su esseri umani e animali.

\section{Bibliografia}

1. Cappelletti P (2018) Medicina 4.0. Un'introduzione. Riv Ital Med Lab 14:131-135

2. https://www.analytica.de/media/website/files/pdf/final-report.pdf (Accesso 12 ottobre 2018)

3. https://elearning.eflm.eu/course/view.php?id=38 (Accesso 12 ottobre 2018)

4. Burtis CA (1996) Converging technologies and their impact on the clinical laboratory. Clin Chem 42:1735-1749

5. Friedman BA (2001) The total laboratory solution: a new laboratory E-business model based on a vertical laboratory meta-network. Clin Chem 47:1526-1535 
6. Price CP (2010) Roots, development and future directions of laboratory medicine. Clin Chem Lab Med 48:903-909

7. King HC, Sinha AA (2001) Gene expression profile analysis by DNA microarrays: promise and pitfalls. JAMA 286:2280-2288

8. Tanaka H (2010) Omics-based medicine and systems pathology. A new perspective for personalized and predictive medicine. Methods Inf Med 49:173-185

9. National Research Council (2011) Toward Precision Medicine: building a knowledge network for biomedical research and a new taxonomy of disease. https://www.nap.edu/catalog/13284/ toward-precision-medicine-building-a-knowledge-network-forbiomedical-research (Accesso 12 ottobre 2018)

10. https://www.mskcc.org/press-releases/msk-impact-first-tumorprofiling-multiplex-panel-authorized-fda-setting-new-pathwaymarket-future-oncopanels (Accesso 12 ottobre 2018)

11. Jani IV, Peters TF (2013) How point-of-care testing could drive innovation in global health. N Engl J Med 368:2319-2324

12. Luppa PB, Müller C, Schlichtiger A et al (2011) Point-of-care testing (POCT): Current techniques and future perspectives. Trends in Analytical Chemistry 30:887-898
13. Busko M (2018) FDA approves artificial pancreas for children with type 1 diabetes. Medscape https://www.medscape.com/ viewarticle/898453 (Accesso 12 ottobre 2018)

14. https://www.leicabiosystems.com/pathologyleaders/what-isdigital-pathology/ (Accesso 12 ottobre 2018)

15. http://eprints.whiterose.ac.uk/120334/1/05Digital\%20 Pathology\%20in\%20Clinical\%20Use.pdf (Accesso 12 ottobre 2018)

16. Päuser S (2018) Illuminating the true nature of diseases. Digit Biomark 2:90-93. https://www.karger.com/Article/FullText/492142 (Accesso 12 ottobre 2018)

17. Tolan NV, Parnas ML, Baudhuin LM et al (2015) "Big Data" in Laboratory Medicine. Clin Chem 61:1433-1440

18. Kricka LA, Polsky TG, Park JY et al (2015) The future of Laboratory Medicine-a 2014 perspective. Clin Chem Acta 438:284-303

19. Topol E (2015) The patient will see you now. The future of Medicine is in your hands. Basic Book, New York

20. Cappelletti P (2015) Il mutamento delle professioni della Medicina di Laboratorio. Riv Ital Med Lab 11:123-131 